\title{
Foxy Methoxy: A New Drug Of Abuse
}

\author{
Susan C. Smolinske, PharmD, DABAT, Rahul Rastogi, MD ${ }^{\mathrm{b}}$, Stephen Schenkel, MD, MPPc \\ a Children's Hospital of Michigan Regional Poison Control Center, Detroit, MI \\ b St. Joseph Mercy Hospital, Ann Arbor, MI \\ c University of Michigan, Ann Arbor, MI
}

REPRINTED FROM THE INT J MED TOXICOL 2004; 7(1): 3.

\begin{abstract}
Introduction: In 1999, a new synthetic tryptamine, 5-MeO-DIPT, became known as a street drug, with the street name of "Foxy" or "Foxy Methoxy". By February 2003, the DEA reported law enforcement seizures and/or reports of abuse in 12 states. We report a case along with an analysis of poison center data on this new drug of abuse.

Case report: A 19-year-old male was brought to the emergency department following ingestion of a larger than his usual dose of Foxy. Upon arrival, he had hallucinations, hypertension, tachycardia, mydriasis, and catalepsy. Symptoms resolved within two hours after administration of lorazepam and he recovered uneventfully.

Discussion: The AAPCC TESS database contained 41 exposures to "Foxy" between April, 2002 and June, 2003; 26 had moderate or major effects, indicating this drug has significant toxic potential. Given the expanding use of this and other club drugs, the spectrum of toxicity from this new agent will continue to be elucidated.
\end{abstract}

\section{INTRODUCTION}

In 1999, a new synthetic tryptamine, 5-methoxy-N, Ndiisopropyltryptamine (5-MeO-DIPT), became known as a street drug, with the street name of "Foxy" or "Foxy Methoxy." By February, 2003, the DEA reported law enforcement seizures and/or reports of abuse in 12 states [1]. We report a case with an unusual neurological presentation.

\section{CASE REPORT}

A 19-year-old male with multiple body piercing was brought to the emergency department at 7 A.M. by his girlfriend. On initial examination he was entirely unable to answer any questions, instead he was staring into space with his eyes open and repeating "yeah ..." in response to any stimulation. His girlfriend revealed that he had taken some "Foxy" - a white powder purchased from an Internet source for $\$ 75 /$ gram at 9 P.M. the prior evening. Seeking a stronger high, he had taken more than ever before, but she was unable to provide the exact quantity. He had no past medical history, took no regular medications, and had no allergies to medications. She did not believe there was any additional drug use.

On examination he had a pulse of $138 / \mathrm{min}$, respiratory rate of $15 / \mathrm{min}$, blood pressure of $191 / 102 \mathrm{~mm} \mathrm{Hg}$, and an oxygen saturation of $100 \%$. His pupils were dilated at $7 \mathrm{~mm}$ and responsive, his skin cool and dry. Lungs were clear, heart sounds regular and hyperdynamic, and abdomen soft with occasional bowel

Note: Presented in part as The 2003 North American Congress of Clinical Toxicology Annual meeting, September 4-9, 2003, Chicago, Illinois.

Keywords: tryptamine, serotonin, hallucinogen, catalepsy, poisoning

Corresponding Author: Susan C. Smolinske, PharmD, DABAT, Children's Hospital of Michigan Regional Poison Control Center, 4160 John R, Suite 616, Detroit, MI 48201. Email: ssmolins@dmc.org 
sounds. He continued to reply "yeah ..." throughout the examination. His extremities demonstrated a waxy plasticity; remaining in whatever position the examiner placed them.

Laboratory studies demonstrated an elevated white blood cell count with a left shift and slightly elevated platelet count. His sodium was $138 \mathrm{mEq} / \mathrm{L}$, potassium $3.6 \mathrm{mEq} / \mathrm{L}$, bicarbonate $23 \mathrm{mg} / \mathrm{dL}$, and glucose $275 \mathrm{mg} / \mathrm{dL}$. Serum blood urea nitrogen and creatinine were unremarkable. Calcium was $9.6 \mathrm{mg} / \mathrm{dL}$ and myoglobin $19.2 \mathrm{mcg} / \mathrm{L}$. An ECG showed sinus tachycardia. Urinalysis demonstrated $3+$ glucose and was otherwise unremarkable. His urine drug screen was positive for cocaine and phencyclidine.

Treatment was symptomatic and supportive. As he was maintaining his airway, no airway intervention was indicated. An intravenous line was placed, as was a Foley catheter. He received normal saline. He was given $1 \mathrm{mg}$ lorazepam twice for his tachycardia and hypertension. Over the next several hours the tachycardia and hypertension resolved, and the patient became more responsive. The patient then denied the use of cocaine and explained that he had never taken so much "Foxy." By noon he was appropriate and comfortable, his heart rate and blood pressure normalized, and he was discharged home from the emergency department. Urine assay for tryptamines was not available at this hospital.

\section{DISCUSSION}

Foxy or Foxy Methoxy is 5-methoxy-N, N-diisopropyltryptamine, a synthetic tryptamine in the same class as psilocybin or psilocin. Law enforcement agencies report seizures of capsules and tablets in ten states since 2001. Its' use has been reported at clubs in Arizona, California, Florida, Delaware, Idaho, and New York, and, as this case suggests, is likely more widely distributed [1]. Foxy is usually administered in tablet form, often described with an alien or spider logo, but powder-filled capsules have been confiscated. This case and one other reported to TESS involved powdered form. The powder and ingredients for manufacture appear still to be available on the Internet, as are instructions regarding its dosing and usage [2]. As in this case, users develop tolerance and often escalate the dose to achieve a greater effect. An emergency scheduling into Schedule I took place on February 28, 2003, [3] however the chemical is still listed as available "for scientific purposes" on at least one web site [2].

The most detailed descriptions of the effects of the drug are those of its developers who synthesized it along with other tryptamines [4]. They found a threshold for hallucinogenic activity at $4 \mathrm{mg}$ with an effective range from $6-10 \mathrm{mg}$. Time of onset was 20-30 minutes and peak effect at 1-1.5 hours, with resolution of symptoms by approximately six hours. In their initial report, Shulgin and Carter felt that the drugs were relatively free of autonomic side-effects or any indicators of toxicity beyond mild nausea and muscular hyperreflexia.

Shulgin and Shulgin also described the use of the drugs in more poetic tones in a book published on the Internet. At six mg they noted "What remains with me the longest was the awareness of vibrations, and what felt best was the stillness" [5]. A $10 \mathrm{mg}$ dose, though, did not necessarily lead to a pleasant experience: "As I became more turned on the greater I felt the sense of hypertension. A mind/body load became uncomfortable. It was never psychedelic in the way of acid or psilocybin. My muscles, gluteus maximus, the lateral rotators that connect to the trochanter and the large muscles that connect to the hamstrings, all contracted and spasmed."

There is little guidance as to the side effects or toxic effects of 5-MeO-DIPT. The general side effects of the tryptamine class partially explain the symptoms of our case patient, specifically his hallucinations, mydriasis, hypertension, and tachycardia. A 17year-old patient who ingested a related compound purchased over the Internet, alpha-methyltryptamine, exhibited bizarre behavior, tachycardia, diaphoresis, and mydriasis, with an onset of 15 minutes after ingestion [6]. A 21-year-old patient who ingested a "Foxy" pill purchased on the street and confirmed with urine GCMS developed visual hallucinations, mydriasis, and temporary paralysis of his extremities lasting 3.5 hours. Notable is this case and in our patient, the urine immunoassay was negative for amphetamines [7].

The patient's soft pliability was the most remarkable feature of his presentation, yet is not a finding typical of the tryptamines. Such a presentation suggests effects of other intoxicants including LSD, lithium, monoamine oxidase inhibitors, or side effects such as neuroleptic malignant syndrome or serotonin syndrome. Rigidity has been described as an effect of phencyclidine; our patient, however, was distinctly more waxy and plastic than rigid [8]. Our patient had no temperature recorded to suggest whether there was associated hyperthermia. The positive urine drug screen for phencyclidine may represent a class-based cross-reaction from 5-MeO-DIPT, although there are no data regarding cross-reaction with tryptamines. It is also possible the patient co-ingested dextromethorphan, which is a common cross-reactant in this geographic area, or that the purchased product contained cocaine and/or PCP as an adulterant. The combined effects of 5-MeO-DIPT and dextromethorphan could result in serotonin syndrome, as the tryptamines are serotonin agonists. Unfortunately, a confirmatory assay was not available. The pharmacology and receptor affinities of 5-MeO-DIPT have not been studied. Catalepsy has been speculated to occur from 5HT2 agonists, as a result of associated decreased cortical dopamine levels.

The positive cocaine screen is somewhat more difficult to explain. The patient later denied any recent use of cocaine, but the sympathomimetic effects of the drug would also explain his tachycardia and hypertension. The combined use might also exacerbate the muscular plasticity due to serotinergic effects of cocaine. It is conceivable that our patient did not know about cocaine lacing his 5-MeO-DIPT powder, but more likely he denied use of illicit substances to avoid arrest. He maintained that 5-MeO-DIPT was legal and a research chemical. We cannot fully explain the patient's glucosuria and hyperglycemia. He was not 


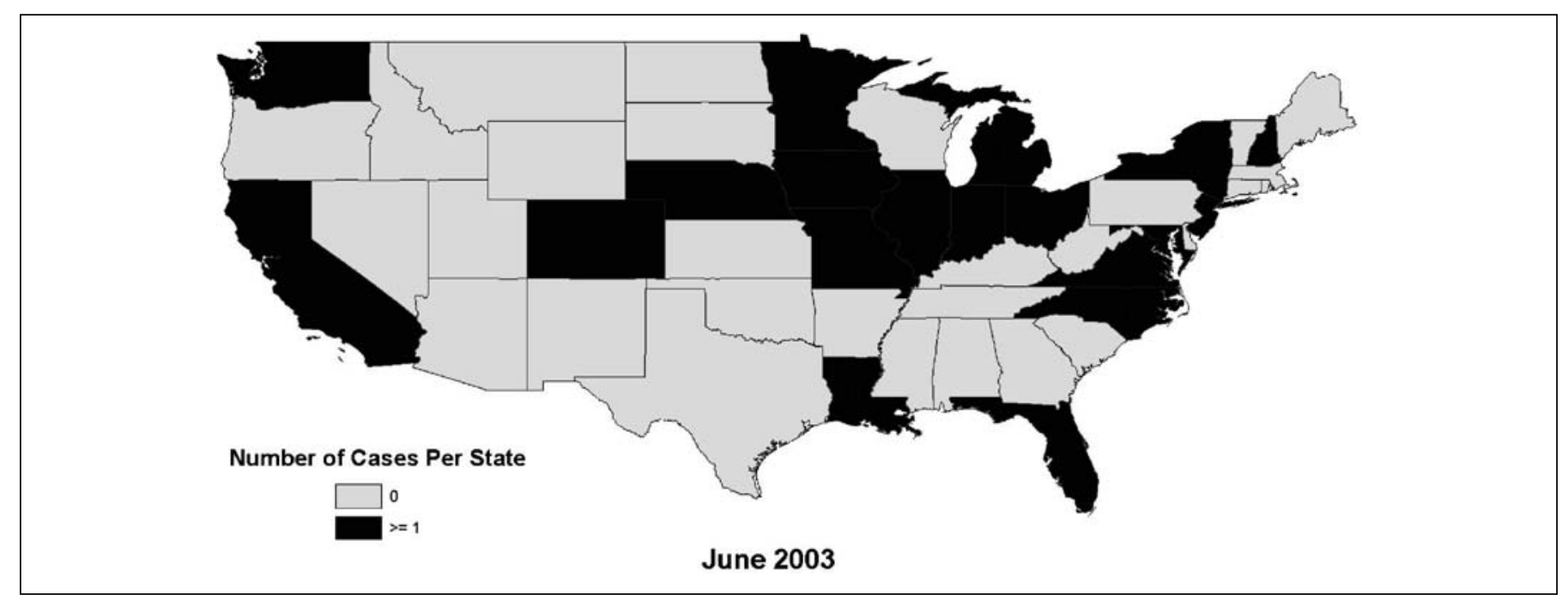

Figure 1. Cumulative cases of 5 methoxy di isopropyl tryptamine (abuse, suicide) by month, by state, April $2002-J u n e ~ 2003$. Acknowledgement: Map created by Amy B. Funk, U.S. Centers for Disease Control.

diabetic. He received no glucose containing fluids. This could have arisen from his stress reaction and hyperdynamic state.

The American Association of Poison Control Centers TESS database was searched, and contained 41 cases of 5-MeO-DIPT exposure reports to poison centers from April 2002, which was the earliest year the term could be searched, to the end of June 2003. The outcome of these exposures was no effect in one case, minor in 8 , moderate in 26 , and major in two. Clinical effects commonly involved agitation (59\%), hallucinations (39\%), tachycardia (37\%), hypertension (17\%), and confusion (15\%). Tremors (5\%) and seizures ( 1 case) were rare. Traclomg of cumulative cases indicates a relatively constant low rate of reporting, indicative of a limited number of suppliers.

However, the geographic area of TESS cases has expanded (Figure 1). Only three states were involved from April 2002 to July 2002, then seven by the end of October, then 12 by December 2002 , with our case representing one of two new states reporting a case in the first two months of 2003, ending in 17 states by June 2003. In addition, there were two states where information calls about 5-MeO-DIPT were received by a poison center, but no human exposures reported. Such plots could be monitored prospectively and in real-time, providing an early warning should abuse begin to increase. Often scheduling a drug as a controlled substance has the effect of stimulating desire for abuse, and this could easily be demonstrated through TESS monitoring. Other surveillance systems, such as DAWN, are less currently updated, with the latest reports only for the year 2001, and no mention of "Foxy."

We believe that this is the first case of catalepsy described with this agent, with one previous report of limb "paralysis" [7]. The duration of more than 5 hours was longer than that previously reported, possibly due to the large dose or interaction with possible co-ingestants. Given the expanding use of this and other club drugs, the spectrum of toxicity from this new agent will continue to be elucidated.

\section{Acknowledgment}

We are grateful to William Watson, PharmD and the American Association of Poison Control Centers for analysis of the TESS database.

\section{REFERENCES}

1. DEA Intelligence Division. Trippin? on Tryptamines: The Emergence of Foxy and AMT as Drugs of Abuse. Microgram Bulletin 2002;35:264-268.

2. Direct NIC [homepage on the Internet], [under construction; cited 1/5/04]. Available from: http://www.edtchemical .com/5-MeO-DIPT.htm.

3. Drug Enforcement Administration. Schedules of Controlled Substances:Temporary Placement of Alphamethyltryptamine and 5-methoxy-N,N-diisopropyltryptamine Into Schedule I. Federal Register 2003:68:4127-4130.

4. Shulgin AT, Carter MF. N,N-Diisopropyltryptamine (DIPT) and 5-Methoxy-N,N-Diisopropyltryptamine (5-MeO-DIPT. Two Orally Active Tryptamine Analogs with CNS Activity. Communications in Psychopharmacology 1980; 4:363-369.

5. Shulgin A, Shulgin A. PIHKAL: A Chemical Love Story. Berkeley: Transform Press; 1991.

6. Long H, Nelson LS, Hoffman RS. Alpha-methyltryptamine: Revisited via easy internet access. Vet Human Toxicol 2003; $45: 149$.

7. Meatherall R, Sharma P. Foxy, a designer tryptamine hallucinogen. J Anal Toxicol 2003; 27:313-317.

8. Olson KR. Phencyclidine. In: Olson KR, editor. Poisoning and drug overdose. $3^{\text {rd }}$ ed. Stamford: Appleton \& Lange; 1999. p. 254-255. 